\title{
Prevalencia de desnutrición en pacientes con enfermedad renal crónica terminal en un hospital nacional de Lima, Perú
}

\section{Prevalence of malnutrition in patients with terminal chronic kidney disease in a national hospital in Lima, Peru}

\author{
Yveth Munive-Yachachi ${ }^{1, a}$, Doris Delgado-Pérez ${ }^{2, b}$ \\ ${ }^{1}$ Unidad de Posgrado, Facultad de Medicina, Universidad Nacional Mayor de San Marcos. Lima, Perú. \\ ${ }^{2}$ Centro de Investigación de Bioquímica y Nutrición, Universidad Nacional Mayor de San Marcos. Lima, Perú. \\ ${ }^{a}$ Maestrando de nutrición con mención en nutrición clínica, ORCID: https://orcid.org/0000-0002-9535-8165 \\ ${ }^{b}$ Doctor en ciencias de la salud, ORCID: https://orcid.org/ 0000-0001-5949-754X
}

An Fac med. 2021;82(1):21-6. / DOI: https://doi.org/10.15381/anales.v82i1.18037.

\begin{abstract}
Correspondencia:
Yveth Munive

yvethmunive@gmail.com

Recibido: 13 de marzo 2021

Aprobado: 11 de junio 2021

Publicación en línea: 3 de julio 2021

Conflictos de interés: Las autoras declaran no tener conflictos de interés.
\end{abstract}

Fuente de financiamiento: Autofinanciado

Citar como: Munive-Yachachi Y, DelgadoPérez D. Prevalencia de desnutrición en pacientes con enfermedad renal crónica terminal en un hospital nacional de Lima, Perú. An Fac med. 2021;82(1): 21-6. DOI: https:/doi.org/10.15381/anales. v82i1.18037.

\section{Resumen}

Introducción. La enfermedad renal crónica (ERC) es un problema de salud pública a nivel mundial que se caracteriza por un deterioro persistente y progresivo de la función renal. En etapa avanzada es necesaria la terapia de reemplazo renal que desencadena una serie de trastornos metabólicos y nutricionales. Objetivo. Determinar la prevalencia de desnutrición en pacientes con enfermedad renal crónica terminal atendidos en un hospital nacional en Lima, Perú. Método. Se realizó un estudio descriptivo en pacientes con ERC, mediante entrevistas y mediciones antropométricas. Se utilizó el score de malnutrición e inflamación (MIS) para la evaluación del estado nutricional. Resultados. Se evaluaron 155 pacientes con ERC terminal, 106 $(68,4 \%)$ fueron varones. La prevalencia de desnutrición fue de $36 \%$; y para el síndrome de desgaste proteico energético fue de $74 \%$. Conclusiones. Se encontró un porcentaje considerable de pacientes desnutridos, que podria ser consecuencia de diversos factores que convergen en esta patología; las más vulnerables al desarrollo de este sindrome complejo fueron las mujeres.

Palabras clave: Evaluación Nutricional; Insuficiencia Renal Crónica; Hemodiálisis; Desnutrición; Perú (fuente: DeCS BIREME).

\section{Abstract}

Introduction. Chronic kidney disease (CKD) is a global public health problem characterized by a persistent and progressive deterioration of kidney function. In advanced stage, renal replacement therapy is necessary, which triggers a series of metabolic and nutritional disorders. Objective. To determine the prevalence of malnutrition in patients with terminal chronic kidney disease treated at a national hospital in Lima, Peru. Method. A descriptive study was carried out in patients with CKD, through interviews and anthropometric measurements. The malnutrition and inflammation score (MIS) was used to assess nutritional status. Results. 155 patients with terminal CKD were evaluated, 106 (68.4\%) were men. The prevalence of malnutrition was $36 \%$; and for the protein-energy wasting syndrome it was $74 \%$. Conclusions. A considerable percentage of malnourished patients was found, which could be the consequence of various factors that converge in this pathology; the most vulnerable to the development of this complex syndrome were women.

Keywords: Nutrition Assessment; Renal Insufficiency, Chronic; Renal Dialysis; Malnutrition; Peru (source: MeSH NLM). 


\section{INTRODUCCIÓN}

La enfermedad renal crónica (ERC) es considerada un problema de salud pública que afecta a más del 10\% de la población a nivel mundial (1). En el Perú, según datos del año 2014, el 13\% de la población adulta (mayor de 20 años) tenía ERC en pre-diálisis y en etapa terminal 19197 personas $(0,1 \%)^{(2)}$. Esta enfermedad se caracteriza por un deterioro persistente y progresivo de la función renal y en etapas avanzadas es necesaria la terapia de reemplazo renal (TRR) como la hemodiálisis, diálisis peritoneal y trasplante renal ${ }^{(3)}$.

La ERC y la TRR conducen a trastornos metabólicos y nutricionales que consisten en emaciación proteica energética, sarcopenia e inflamación, factores considerados como los más importantes de muerte ${ }^{(4)}$. Por ello es necesario mantener un buen estado nutricional y sobre todo en pacientes que se someten a hemodiálisis de mantenimiento ${ }^{(4,5,6)}$. En estos pacientes la prevalencia de desnutrición varía entre el $10 \%$ al $70 \%$ según el método de diagnóstico utilizado e incluso un $40 \%$ de pacientes que inician la TRR presentan síntomas de desnutrición ${ }^{(7,8)}$. Se estima que en una sesión de hemodiálisis hay una disminución de 4-9 g de aminoácidos libres, de 2-3 g de polipéptidos, vitaminas hidrosolubles (B1, B6, C, B9, B8, B5), carnitina y oligoelementos. Esto debido al uso de membranas de alta permeabilidad, extracciones sanguíneas, venopunción, pérdidas en las líneas de diálisis y el dializador ${ }^{(9)}$.

La evaluación del estado nutricional permite conocer los factores desencadenantes de la desnutrición, identificar pacientes en riesgo y planificar el tratamiento nutricional ${ }^{(9)}$. En la práctica clínica existen métodos subjetivos como la escala de malnutrición inflamación (MIS) por sus siglas en inglés (Malnutrition Inflammation Score), herramienta integral, cuantitativa y sencilla para aplicar en hemodiálisis; además, presenta asociaciones significativas con variables antropométricas e inflamatorias ${ }^{(10,11,12)}$. Estos métodos de cribado nutricional son válidos para identificar la presencia de riesgo nutricional, siendo necesario luego una evaluación completa que incluya métodos objetivos de antropometría, marcadores bioquímicos e inflamatorios ${ }^{(9)}$.
En diversos países se utiliza el MIS, como en Emiratos Árabes Unidos, donde se reportó 34\% de desnutrición en 70 pacientes en hemodiálisis (PHD) ${ }^{(13)}$, el MIS se correlacionó con varios sustitutos de la composición corporal, calidad de vida y riesgo de muerte ${ }^{(10)}$. En Corea se reportó entre $31 \%$ a $49 \%$ con desnutrición en 145 pacientes en hemodiálisis ${ }^{(7)}$. Otro estudio en México reportó desnutrición de $54 \%$ en 128 pacientes en hemodiálisis (14). En el Perú, en la ciudad de Chiclayo, se observó $61 \%$ de desnutrición en 99 pacientes en hemodiálisis, donde el MIS presentó mayor relación con circunferencias antropométricas ${ }^{(15)}$. Estos estudios demuestran la utilidad de esta herramienta para evaluar la presencia y las implicaciones del síndrome de desgaste proteico energético ${ }^{(14)}$.

En nuestro país no se conoce la prevalencia de desnutrición en pacientes con ERC terminal en hospitales públicos; es preciso evaluar un nuevo grupo objetivo, para incentivar el desarrollo de protocolos de prevención que permitan una intervención nutricional oportuna que dismuya el riesgo de mortalidad por esta condición. Así, el objetivo del presente estudio fue determinar la prevalencia de desnutrición en adultos con enfermedad renal crónica terminal en un hospital nacional de Lima, Perú.

\section{MÉTODOS}

\section{Diseño del estudio}

Se realizó un estudio observacional, descriptivo y transversal ${ }^{(16)}$, en los tres centros de hemodiálisis dependientes del Departamento de Nefrología del Hospital de la Policía Nacional Del Perú Luis N. Sáenz, durante el mes de agosto 2019.

\section{Población y muestra}

La población estuvo constituida por 256 pacientes adultos ambulatorios de ambos sexos con enfermedad renal crónica terminal en hemodiálisis. Todos los pacientes fueron de atención ambulatoria en los tres centros de hemodiálisis. Estos centros fueron Santa Ana, Servimedic y San Ignacio de Loyola, pertenecientes al Departamento de Nefrología del Hos- pital de la Policía Nacional del Perú Luis N. Sáenz de la ciudad de Lima.

El tamaño de muestra se obtuvo mediante la fórmula para estudios descriptivos, variable de tipo cualitativa para población finita ${ }^{(17)}$, con $95 \%$ de nivel de confianza y un error de $5 \%$, obteniendose una muestra total de 155 pacientes. Se estimó una muestra proporcional por cada centro de hemodiálisis. Se calculó para el centro de hemodiálisis "Santa Ana" 55 pacientes, para "Servimedic" 49 pacientes y para "San Ignacio de Loyola" 51 pacientes.

El muestreo fue por conveniencia hasta cubrir la cantidad estimada para ese centro. Se excluyeron del estudio a pacientes con tratamiento oncológico o que se encontraban hospitalizados.

\section{Variable estado nutricional}

Para determinar la valoración del estado nutricional en los pacientes ambulatorios con hemodiálisis se utilizó el método de cribado nutricional con la herramienta MIS. Este método fue considerado idóneo para este tipo de pacientes por ser práctico, rápido y eficaz para detectar la desnutrición ${ }^{(10)}$.

Se utilizó un instrumento validado MIS ${ }^{(18)}$ y un formato de datos de filiación. El MIS estuvo dividido en 4 secciones: a) la valoración global subjetiva con 5 criterios (cambio de peso en los últimos 3 a 6 meses; cambio en la ingesta dietética; síntomas gastrointestinales como anorexia, emesis y enfermedad diarreica; deterioro en la capacidad funcional relacionada con factores nutricionales; y comorbilidades que incluye la cantidad de años en diálisis). b) exploración física para detectar pérdida de grasa subcutánea y pérdida de masa muscular. c) determinación del índice de masa corporal $\left(\mathrm{Kg} / \mathrm{m}^{2}\right)$. d) marcadores bioquímicos de albúmina y transferrina. La toma de medidas antropométricas se realizó después de la sesión de diálisis.

La escala para determinar la desnutrición fue la siguiente, normal: 0 puntos, desnutrición leve 1-9 puntos, desnutrición moderada 10-19 puntos, desnutrición grave 20-29 puntos y desnutrición severa 30 puntos. Para identificar el síndrome de desgaste proteico energético (PEW), por sus siglas en inglés (Protein 
Energy Wasting), se utilizó un puntaje del MIS $>7^{(19,20)}$.

\section{Análisis estadístico}

Después de revisar las encuestas fueron tabuladas en el programa Excel 2013. Los nombres de los participantes en el estudio fueron reemplazados por códigos. Para el análisis estadístico se utilizó el software SPSS versión 20. Las variables cualitativas categóricas se presentaron con frecuencias. Las variables continuas se presentaron como promedios y desviación estándar. El análisis inferencial se utilizó para determinar la correlación entre el grado de desnutrición con el género y la edad aplicando la prueba chicuadrado, teniendo un nivel de confianza del 95\%, con un error máximo del 5\%.

\section{Consideraciones éticas}

El estudio fue aprobado por el Comité de Ética en Investigación de la Facultad de Medicina de la Universidad Nacional Mayor de San Marcos (Acta N 19-0024). Previo a la recolección de datos se explicó en detalle el estudio a cada paciente para lograr una participación voluntaria y se les solicitó el correspondiente consentimiento informado.

\section{RESULTADOS}

Se evaluaron a un total de 155 pacientes adultos con enfermedad renal crónica terminal (ERCT). La edad promedio fue de 63,5 $\pm 10,7$ años. El puntaje medio de MIS fue 8,8 ( \pm ) 3,4; valor clasificado como desnutrición leve.

Según el grupo de edad, un tercio presentó entre 50 a 59 años y más de la mitad de los pacientes fueron adultos mayores con más de 60 años. Las causas que ocasionaron el desarrollo de la ERCT fueron la diabetes mellitus tipo 2 con mayor proporción, seguida de la hipertensión arterial. Un tercio de los pacientes presentó ambas comorbilidades, tal como se muestra en la tabla 1.

El score MIS evaluó el estado nutricional a través de 10 indicadores, de los cuáles se observó que tuvo mayor grado de severidad el "nivel de transferrina" debido a que más de un tercio presentó menos de 140 mg/dL; 8 de cada 10 pa- cientes tuvieron valores de moderado y severamente disminuidos.

Por otro lado, dentro de los indicadores positivos, más de la mitad de los pacientes no refirió pérdida de peso entre los últimos 3 a 6 meses y la misma proporción mencionó mantener buen apetito, tal como se muestran en la tabla 2.

El 36\% de pacientes presentó desnutrición moderada a grave y más de la mitad de los pacientes tuvieron un grado de desnutrición leve, como se muestra en la figura 1. Además, se encontró un $73,5 \%$ (114) de PEW y 26,5\% (41) sin PEW. Según género, se observó que fue mayor la prevalencia con desnutrición moderada en mujeres que en varones $p=0,027(p<0,05)$. Por otro lado, más de la mitad de los pacientes varones presentó desnutrición leve, superando a las mujeres, como se muestra en la tabla 3.

El rango de edad entre 41 a 55 años fue el grupo con mayor prevalencia de desnutrición leve; desnutrición moderada se presentó con un porcentaje equitativo en los pacientes menores de 40 años y mayores de 71 años $p=0,126(p<0,05)$. Tabla 3.

\section{DISCUSIÓN}

El estudio determinó la prevalencia de desnutrición en pacientes con enfer- medad renal crónica terminal en terapia de hemodiálisis. El desencadenante de la desnutrición no se puede atribuir a ningún factor único, sino como consecuencia de una serie de factores propios de la diálisis, diversas comorbilidades, alteraciones metabólicas y hormonales, insulino resistencia, inflamación y hospitalizaciones frecuentes ${ }^{(5)}$. El estado nutricional es considerado un factor independiente de mortalidad, enfoque que se ha relacionado en estudios previos. Se encontró un porcentaje significativo de pacientes con desnutrición moderada, información que servirá para posteriores políticas de prevención.

Este estudio encontró que más de la mitad de los pacientes fueron adultos mayores con una edad media de 64 años. Semejante a los datos de nuestro país donde se prevé un incremento exponencial en $21,8 \%$ de población mayor de 60 años para el año $2050^{\text {(2) }}$. Considerado uno de los determinantes de la ERCT debido a que las principales patologías que aqueja a esta población son de tipo crónico degenerativas y es coherente con la disminución de la tasa de filtrado glomerular (TFG) con la edad (2).

Se ha identificado que la DM e HTA constituyen un $70,5 \%$ de las causas de ERC en el Perú ${ }^{(2)}$, coincidiendo con los resultados de nuestro estudio que presentó a la DM como principal causante de la enfer-

Tabla 1. Características de los pacientes adultos con ERCT. Hospital Luis N. Sáenz 2019, Lima, Perú $(n=155)$

\begin{tabular}{lcccc} 
Características & \multicolumn{2}{c}{$\begin{array}{c}\text { Masculino } \\
\text { (n= 106) }\end{array}$} & \multicolumn{2}{c}{$\begin{array}{c}\text { Femenino } \\
\text { (n= 49) }\end{array}$} \\
\hline Grupo de edad & $n$ & $\%$ & $n$ & $\%$ \\
\hline$<40$ años & 1 & 0,9 & 3 & 6,1 \\
\hline $41-55$ años & 11 & 10,4 & 10 & 20,4 \\
\hline $56-70$ años & 69 & 65,1 & 21 & 42,9 \\
\hline$>71$ años & 25 & 23,6 & 15 & 30,6 \\
\hline Causas de la ERCT & & & & \\
\hline Diabetes Mellitus 2 (DM) & 47 & 44,3 & 18 & 36,7 \\
\hline Hipertensión Arterial (HTA) & 35 & 33,0 & 10 & 20,4 \\
\hline DM e HTA & 11 & 10,3 & 8 & 16,3 \\
\hline Síndrome Nefrótico & 1 & 0,9 & 0 & 0,0 \\
\hline Otros & 7 & 6,6 & 7 & 14,2 \\
\hline Desconoce & 5 & 4,7 & 6 & 12,2 \\
\hline
\end{tabular}




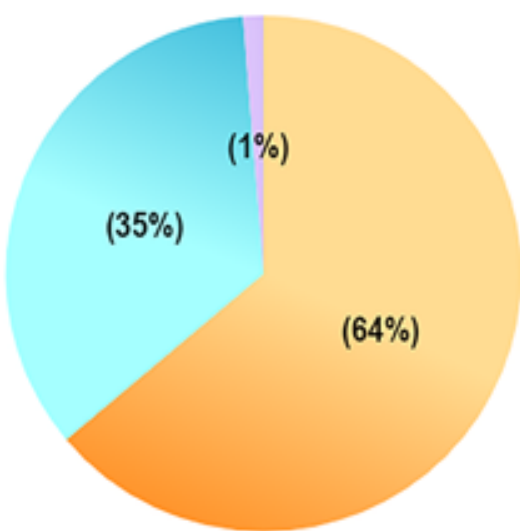

Desnutrición leve Desnutrición moderada Desnutrición grave

Figura 1. Prevalencia de desnutrición con el score MIS (Malnutrition Inflammation Score), en pacientes adultos con ERCT. Hospital Luis N. Sáenz 2019, Lima, Perú (n=155).

medad. La HTA es considerada como causa y consecuencia de la enfermedad renal, diversos estudios indican que la elevación de la presión arterial sistólica más que diastólica es un factor independiente para desarrollar esta enfermedad ${ }^{(2,21,22)}$.

En la práctica clínica existen diferentes métodos para determinar la preva- lencia de desnutrición que pueden ser subjetivos y objetivos. En el presente estudio se utilizó el MIS como una herramienta subjetiva para valorar el estado nutricional, puesto que es la más indicada para pacientes en hemodiálisis y la evidencia científica demostró asociaciones significativas con parámetros antropométricos e inflamatorios ${ }^{(10,13,20)}$.
Un tercio de los pacientes (36\%) presentó desnutrición moderada - grave y más de la mitad (64\%) tuvieron desnutrición leve. No obstante, un estudio transversal realizado en pacientes con ERCT en hemodiálisis de la ciudad de Chiclayo (Perú), reportó una prevalencia de 61\% con desnutrición ${ }^{(15)}$. En cambio, un estudio transversal en México y otro estudio longitudinal en Brasil reportaron una prevalencia en la mitad de los pacientes 53\% y $50 \%$ con desnutrición. Por otro lado, un estudio transversal efectuado en Malasia, país desarrollado, detectó una prevalencia de $88 \%$ con desnutrición, esto podría explicarse por los diferentes puntos de corte empleados para el MIS ${ }^{(14,23,24)}$.

Por otra parte, dos estudios transversales ejecutados en Emiratos Árabes Unidos y España países desarrollados, encuentran una prevalencia de $48 \%$ y $46 \%$ con desnutrición moderada, valores que se aproximan al presente estudio dado que utilizan el mismo punto de corte y los criterios de inclusión son similares ${ }^{(13,25)}$.

Los resultados obtenidos en el presente estudio con el MIS permiten se-

Tabla 2. Componentes del score MIS (Malnutrition Inflammation Score), en pacientes adultos con ERCT. Hospital Luis N. Sáenz 2019, Lima, Perú $(n=155)$

\begin{tabular}{|c|c|c|c|c|}
\hline Componentes del score MIS & $\begin{array}{c}\text { Normal } \\
n(\%)\end{array}$ & $\begin{array}{l}\text { Leve } \\
\mathrm{n}(\%)\end{array}$ & $\begin{array}{l}\text { Moderado } \\
n(\%)\end{array}$ & $\begin{array}{c}\text { Severo } \\
n(\%)\end{array}$ \\
\hline \multirow{2}{*}{$\begin{array}{l}\text { Cambio en peso neto tras diálisis } \\
\text { últimos } 3 \text { a } 6 \text { meses }\end{array}$} & Ningún descenso o $<0,5 \mathrm{~kg}$ & $>0,5-<1 \mathrm{~kg}$ & $>1 \mathrm{~kg}<5 \%$ & $>5 \%$ \\
\hline & $104(64 \%)$ & $22(14 \%)$ & $27(17 \%)$ & $2(1 \%)$ \\
\hline \multirow[t]{2}{*}{ Ingesta dietética } & Buen apetito & $\begin{array}{c}\text { Ingesta de bajo de lo } \\
\text { óptimo }\end{array}$ & Descenso generalizado & Ingesta líquida \\
\hline & $101(65 \%)$ & $41(26 \%)$ & $12(8 \%)$ & $1(1 \%)$ \\
\hline \multirow{2}{*}{ Síntomas gastrointestinales } & Sin síntomas & Síntomas leves & Vómitos ocasionales & Severa anorexia \\
\hline & $95(61 \%)$ & $41(27 \%)$ & $17(11 \%)$ & $2(1 \%)$ \\
\hline \multirow[t]{2}{*}{ Capacidad funcional } & Se siente bien & Dificultad ocasional & $\begin{array}{c}\text { Dificultad con actividades } \\
\text { autónomas }\end{array}$ & $\begin{array}{c}\text { Permanece en cama o } \\
\text { sentado }\end{array}$ \\
\hline & $95(61 \%)$ & $18(12 \%)$ & $24(16 \%)$ & $18(12 \%)$ \\
\hline \multirow{2}{*}{ Comorbilidades -Años en diálisis } & Menos de 1 año & De 1-4 años & Más de 4 años & Comorbilidad severa \\
\hline & $33(21 \%)$ & $82(53 \%)$ & $40(26 \%)$ & 0 \\
\hline Depósitos grasos disminuidos & $14(9 \%)$ & $94(61 \%)$ & 45 (29\%) & $2(1 \%)$ \\
\hline Pérdida de masa muscular & $1(1 \%)$ & $109(70 \%)$ & $41(27 \%)$ & $4(3 \%)$ \\
\hline \multirow{2}{*}{ Índice de Masa Corporal } & $>=20 \mathrm{~kg} / \mathrm{mt}^{2}$ & $18-19,9 \mathrm{~kg} / \mathrm{mt}^{2}$ & $16-17,9 \mathrm{~kg} / \mathrm{mt}^{2}$ & $=<16 \mathrm{~kg} / \mathrm{mt}^{2}$ \\
\hline & $139(90 \%)$ & $13(8 \%)$ & $2(1 \%)$ & $1(1 \%)$ \\
\hline \multirow{2}{*}{ Albúmina sérica } & $>=4 \mathrm{~g} / \mathrm{dl}$ & $3,5-3,9 \mathrm{~g} / \mathrm{dl}$ & $3-3,4 \mathrm{~g} / \mathrm{dl}$ & $=<3 \mathrm{~g} / \mathrm{dl}$ \\
\hline & $86(56 \%)$ & $50(32 \%)$ & $16(10 \%)$ & $3(2 \%)$ \\
\hline \multirow{2}{*}{ Transferrina sérica } & $>=200 \mathrm{mg} / \mathrm{dl}$ & $170-199 \mathrm{mg} / \mathrm{dl}$ & $140-169 \mathrm{mg} / \mathrm{dl}$ & $<140 \mathrm{mg} / \mathrm{dl}$ \\
\hline & $4(3 \%)$ & $19(12 \%)$ & 67 (43\%) & 65 (42\%) \\
\hline
\end{tabular}


Tabla 3. Prevalencia de desnutrición según edad y género, en pacientes adultos con ERCT. Hospital Luis N. Sáenz 2019, Lima, Perú ( $n=155)$.

\begin{tabular}{|c|c|c|c|c|c|c|c|}
\hline \multirow[t]{2}{*}{ Características } & \multicolumn{2}{|c|}{$\begin{array}{c}\text { Desnutrición } \\
\text { Leve }\end{array}$} & \multicolumn{2}{|c|}{$\begin{array}{c}\text { Desnutrición } \\
\text { Moderada }\end{array}$} & \multicolumn{2}{|c|}{$\begin{array}{c}\text { Desnutrición } \\
\text { Grave }\end{array}$} & \multirow[t]{2}{*}{$\begin{array}{c}\text { Chi - } \\
\text { cuadrado } \\
\text { Valor } p\end{array}$} \\
\hline & $n$ & (\%) & $n$ & $(\%)$ & $n$ & (\%) & \\
\hline \multicolumn{8}{|l|}{ Género } \\
\hline Femenino & 26 & 53,06 & 21 & 42,86 & 2 & 4,08 & 0,027 \\
\hline Masculino & 73 & 68,87 & 33 & 31,13 & 0 & 0,00 & \\
\hline \multicolumn{8}{|l|}{ Grupo de edad } \\
\hline$<40$ años & 2 & 50,00 & 2 & 50,00 & 0 & 0,00 & 0,126 \\
\hline 41-55 años & 18 & 85,71 & 3 & 14,29 & 0 & 0,00 & \\
\hline 56-70 años & 59 & 65,56 & 30 & 33,33 & 1 & 1,11 & \\
\hline > 71 años & 20 & 50,00 & 19 & 47,50 & 1 & 2,50 & \\
\hline
\end{tabular}

ñalar que es una herramienta diseñada exclusivamente para pacientes en hemodiálisis. Se utilizó en diversos estudios comparándolo con marcadores bioquímicos, parámetros antropométricos y bioimpedancia. Todos concluyeron que es la mejor herramienta subjetiva, para el diagnóstico de desnutrición e iniciar la evaluación objetiva con la finalidad de reducir la morbi mortalidad en este grupo de pacientes ${ }^{(13,15)}$.

Los pacientes con ERCT cursan con inflamación crónica que se origina por predisposición genética, pero también se debe a la terapia de hemodiálisis y disminución de la TFG. Generando una caquexia urémica y disminución de la masa grasa con el transcurso del tiempo. Así, existe una asociación entre la desnutrición e inflamación que generó la descripción del síndrome de desgaste proteico energético (PEW) ${ }^{(26)}$

En recientes investigaciones se ha reportado que el MIS predice el estado nutricional e inflamatorio igual a la concentración sérica de interluquina-6 (IL-6) e incluso más alta que la proteína $c$ reactiva (PCR). El score MIS contiene la mayoría de criterios principales sugeridos por la Sociedad Internacional de Nutrición y Metabolismo para diagnosticar el PEW (20). Un estudio realizado en pacientes con ERCT en hemodiálisis de México, detectó el PEW en 39\% ${ }^{(19)}$. Respecto a lo anterior, en nuestro estudio encontró el PEW en $74 \%$, lo que podría explicarse porque el tamaño de la muestra fue mayor.
La prevalencia en ambos resultados fue elevada, más aún para el PEW con una diferencia de 39\%. En el Perú no contamos con datos que revelen la prevalencia de desnutrición y en los centros de diálisis aún no se incorpora este tipo de cribado nutricional como el MIS que es de uso habitual en otros países. En consecuencia, la terapia nutricional óptima es una estrategia a veces olvidada en este grupo de pacientes ${ }^{(5)}$.

Se encontró que el género se relaciona con el grado de desnutrición $p=0,027$, siendo el femenino el grupo más afectado con un $(42,8 \%)$ de desnutrición moderada en comparación a los varones con $(31,1 \%)$. Por otro lado, la edad no guarda relación con el desarrollo de la desnutrición $p=0,126$, debido a que los porcentajes fueron equitativos tanto para adultos menores de 40 años $(50,0 \%)$ y mayores de 71 años (47,8\%). Al respecto dos estudios transversales ejecutados en pacientes con ERCT de España e Irán, evidenciaron mayor proporción de mujeres con desnutrición que varones. La edad es un factor de riesgo controvertido ya que no hay una relación significativa que lo vincule con el desarrollo de la desnutrición, acorde con lo encontrado en este estudio ${ }^{(27,28)}$.

La transferrina sérica es un marcador bioquímico que valora el estado nutricional como indicador de la masa proteica visceral. Se le reconoce como uno de los mejores marcadores proteicos, porque es más sensible a su deficiencia y cambia inmediatamente con una dieta inadecua- da ${ }^{(29)}$. Nuestro estudio encontró niveles bajos de transferrina sérica (<200 mg/ $\mathrm{dL}$ ) en $97 \%$ de los pacientes con ERCT y el $42 \%$ de ellos presentaron niveles severamente disminuidos (<140 mg/dl). Esta prevalencia fue similar a un estudio realizado en pacientes con ERCT en hemodiálisis de España. Esto puede deberse a su poder para discernir manifestaciones iniciales de desnutrición corroborando los resultados de este estudio, porque la transferrina es un marcador más precoz que la albúmina en situaciones de desnutrición e incluso inflamación ${ }^{(29)}$.

Por otro lado, la transferrina sérica también es un marcador de utilidad clínica para el monitoreo de la anemia, diversos estudios describieron que los pacientes en diálisis son especialmente vulnerables al desarrollo de anemia ferropénica, a causa de la sangre retenida en la máquina y los túbulos de diálisis; así, existe concordancia con lo encontrado en el estudio ya que el porcentaje con niveles disminuidos fue elevado ${ }^{(30,31)}$.

La anemia es frecuente en los pacientes con ERCT debido al deterioro de la función renal que ocasiona una disminución en la producción de eritropoyetina que se encarga de estimular la producción de glóbulos rojos cuando descienden los niveles de oxígeno. Se describe otras causas como la deficiencia de hierro, inflamación y la acumulación de toxinas urémicas. Lo habitual es que esta complicación aparece en estadio 4 y se agrava conforme progrese la enfermedad ${ }^{(32)}$.

Una limitación importante en el estudio fue la imposibilidad de poder contrastar los resultados de desnutrición o PEW con otros marcadores inflamatorios como el IL-6, PCR y parámetros antropométricos. Además, su naturaleza descriptiva y transversal limita su contribución según los niveles de evidencia, por lo que se debe considerar estudios posteriores para ampliar su impacto en el estado nutricional; sin embargo, contribuye a futuras investigaciones, puesto que no existen referencias previas sobre el estado nutricional en pacientes con ERC terminal en hospitales públicos.

En conclusión, se encontró una prevalencia considerable de pacientes desnutridos con enfermedad renal crónica 
terminal (36\%), que podría ser resultado de diversos factores que convergen en esta patología. La mayor prevalencia se encontró en las mujeres por ser más vulnerables al desarrollo de este síndrome complejo. Los resultados tendrán importancia para la implementación de protocolos de prevención y evaluación nutricional que permitan mejorar los indicadores de mortalidad, en el ámbito de salud pública y privada.

\section{REFERENCIAS BIBLIOGRÁFICAS}

1. Hill N, Fatoba S, Oke J, Hirst J, O'Callaghan C, Lasserson D, et al. Global prevalence of chronic kidney disease - A systematic review and metaanalysis. PLoS ONE. 2016;11(7). DOI: 10.1371/ journal.pone.0158765

2. Dirección General de Epidemiologia [Internet]. Loza C, Ramos W. Análisis de la situación de la enfermedad renal crónica en el Perú, 2015. Lima: Ministerio de Salud; 2016 [citado 29 abril 2021]. Disponible en: https://www.gob.pe/institucion/minsa/informespublicaciones/285012-analisis-de-la-situacion-dela-enfermedad-renal-cronica-en-el-peru-2015

3. Tomonaga Y, Risch L, Szucs T, Ambühl P, Ambuehl $P$. The prevalence of chronic kidney disease in a primary care setting: a Swiss cross-sectional study. PLoS One. 2013;8(7). DOI: 10.1371 / journal. pone.0067848

4. Ikizler TA, Cano N, Franch H, Fouque D, Himmelfarb J, Kalantar-Zadeh K, et al. Prevention and treatment of protein energy wasting in chronic kidney disease patients: a consensus statement by the International Society of Renal Nutrition and Metabolism. Kidney Int. 2013;84(6):1096-1107. DOI: 10.1038/ki.2013.147

5. Ikizler TA. Optimal nutrition in hemodialysis patients. Adv Chronic Kidney Dis. 2013;20(2):181189. DOI: 10.1053/j.ackd.2012.12.002

6. Aggarwal HK, Jain D, Chauda R, Bhatia S, Sehgal R. Assessment of Malnutrition Inflammation Score in Different Stages of Chronic Kidney Disease. Pril (Makedon Akad Nauk Umet Odd Med Nauki). 2018;39(2-3):51-61. DOI:10.2478/ prilozi-2018-0042

7. Lim H, Kim H, Kim J, Park M, Choi S. Nutritional Status and Dietary Management According to Hemodialysis Duration. Clin Nutr Res. 2019;8(1):28-35. DOI:10.7762/cnr.2019.8.1.28

8. Onel M, Garcia M, Andrade L, Pérez J, Martínez R. Evaluación del estado nutricional en pacientes con insuficiencia renal crónica en tratamiento de hemodiálisis. Rev Nefrol Dial Traspl. 2012;32(2):86-5.

9. Serván P, Ruiz I. Nutrition in chronic kidney disease. Nutr Hosp. 2019;36(3):63-69 DOI: 10.20960/nh.02812
10. Rambod M, Bross R, Zitterkoph J, Benner D, Pithia J, Colman S, et al. Association of Malnutrition-Inflammation Score with quality of life and mortality in hemodialysis patients: A 5-year prospective cohort study. Am J Kidney Dis. 2009;53(2):298-309. DOI: 10.1053/j.ajkd.2008.09.018

11. Carrascal S, Codinachs M, Oller L, Pérez E, Juvanteny $\mathrm{E}$, Requena D, et al. Descripción del estado nutricional de los pacientes de una unidad de diálisis mediante el uso de la escala "Malnutrition Inflamation Score". Enferm Nefrol. 2013;16(1):2330. DOI:10.4321/S2254-28842013000100004

12. Japaz M, Amezquita $Y$, Sánchez V, Sánchez C, Fernández E, González-Cutre $P$, et al. Utilidad del Score Malnutrición Inflamación (SMI) en la evaluación nutricional de los pacientes en hemodiálisis. Enferm Nefrol. 2012;15( Suppl 1 ): 80-80.

13. Karavetian M, Salhab N, Rizk R, Poulia KA. Malnutrition-Inflammation Score VS Phase Angle in the Era of GLIM Criteria: A Cross-Sectional Study among Hemodialysis Patients in UAE. Nutrients. 2019;11(11):2771. DOI:10.3390/nu11112771

14. Chávez V, Mejia O, Viveros M, Abraham A, Gutiérrez S, Orizaga C, et al. Prevalencia del sindrome complejo de malnutrición e inflamación y su correlación con las hormonas tiroideas en pacientes en hemodiálisis crónica. Nefrologia. 2018;38(1):57-63. DOI:10.1016/j.nefro.2017.04.003

15. Vásquez-Vargas A. Valor predictivo del estado nutricional de pacientes renales crónicos en hemodiálisis usando índices antropométricos comparado con el score de malnutrición e inflamación. Rev exp med. 2017;3(3):98 - 102.

16. Argimon J, Jiménez J. El proceso de la investigación clinica y epidemiológica. En: Elsevier (Eds): Métodos de investigación clínica y epidemiológica. 4ta edición. España: Fotoletra S.A. 2013:15-20.

17. Aguilar-Barojas, S. Fórmulas para el cálculo de la muestra en investigaciones de salud. Salud en Tabasco. 2005;11(1-2):333-338.

18. Kalantar-Zadeh K, Kopple J, Block G, Humphreys M. A malnutrition-inflammation score is correlated with morbidity and mortality in maintenance hemodialysis patients. Am J Kidney Dis 2001;38(6):1251-63. DOI: 10.5053/ ajkd.2001.29222

19. González A, Correa R, Vázquez A, Vega O, Espinosa C. Relationship between protein-energy wasting in adults with chronic hemodialysis and the response to treatment with erythropoietin. BMC Nephrol. 2019; 20(1). DOI: 10.1186/s12882019-1457-0

20. González A, Arce C, Vega O, Correa R, Espinosa M. Assessment of the reliability and consistency of the "Malnutrition Inflammation Score" (MIS) in Mexican adults with chronic kidney disease for diagnosis of protein-energy wasting syndrome (PEW). Nutr Hosp. 2015;31(3):1352-1358. DOI: 10.3305/NH.2015.31.3.8173

21. Osorio N. Comorbilidades y sobrevida de pacientes con enfermedad renal crónica en terapia hemodiálisis del Centro de Cuidado Renal Davita Barranquilla durante los periodos comprendidos entre el 2012 al 2015 [Tesis de Magister en Salud Pública]. Barranquilla, Colombia: Universidad del Norte, 2017. Disponible en: https://manglar.uninorte.edu.co/handle/10584/7875\#page=1

22. Carrillo R, Bernabé A. Mortalidad por enfermedad renal crónica en el Perú: tendencias nacionales 2003-2015. Rev Peru Med Exp Salud Pública. 2018;35(3):409. DOI: 10.17843/ rpmesp.2018.353.3633

23. Rodrigues J, Santin F, Brito F, Lindholm B, Stenvinkel P, Avesani CM. Nutritional status of older patients on hemodialysis: Which nutritional markers can best predict clinical outcomes?. Nutrition. 2019;65:113-119.DOI:10.1016/j.nut.2019.03.002

24. Harvinder G, Swee W, Karupaiah T, Sahathevan S, Chinna K, Ahmad G, et al. Dialysis Malnutrition and Malnutrition Inflammation Scores: screening tools for prediction of dialysis-related protein-energy wasting in Malaysia. Asia Pac J Clin Nutr. 2016;25(1):26-33. DOI: 10.6133/apjcn.2016.25.1.01

25. Gómez L, Manresa M, Morales J, Garcia E, Robles M, Chevarria J. Estado nutricional del paciente en hemodiálisis y factores asociados. Enferm Nefrol. 2017;20(2):120-125. DOI: 10.4321/s2254288420170000200004

26. Rattanasompattikul M, Molnar M, Zaritsky J, Hatamizadeh P, Jing J, Norris K, et al. Association of malnutrition-inflammation complex and responsiveness to erythropoiesis-stimulating agents in long-term hemodialysis patients. Nephrol Dial Transplant. 2013;28(7):1936-1945. DOI:10.1093/ndt/gfs368

27. Garrido L, Sanz M, Caro C. Variables de la desnutrición en pacientes en diálisis. Enferm Nefrol. 2016; 19 (4): 307-316.

28. Hasheminejad N, Namdari M, Mahmoodi MR, Bahrampour A, Azmandian J. Association of handgrip strength with Malnutrition-Inflammation Score as an assessment of nutritional status in hemodialysis patients. Iran J Kidney Dis. 2016; 10(1):30-35.

29. Palomares M, Oliveras M, Osuna A, Asensio C, Quesada J, López H, et al. Evolución de parámetros bioquímicos nutricionales en pacientes de hemodiálisis durante un año de seguimiento. Nutr Hosp. 2008;23(2):119-125.

30. Cases A, Egocheaga M, Tranche S, Pallarés V, Ojeda R, Górriz J, et al. Anemia en la enfermedad renal crónica: protocolo de estudio, manejo y derivación a Nefrologia [Anemia of chronic kidney disease: Protocol of study, management and referral to Nephrology]. Aten Primaria 2018;50(1):60-64. DOI: 10.1016/j.aprim.2017.09.007

31. Stauffer M, Fan T. Prevalence of anemia in chronic kidney disease in the united states. PLoS One. 2014;9(1). DOI: 10.1371/journal.pone.0084943

32. Gafter-Gvili A, Schechter A, Rozen-Zvi B. Iron deficiency anemia in chronic kidney disease. Acta Haematol. 2019;142(1):44-50. DOI: 10.1159/000496492 